\title{
Entrevista a \\ Myriam Mitjavila
}

Myriam Mitjavila es Trabajadora Social, Doctora en Sociologia por la Universidade de São Paulo (Brasil), Docente e investigadora de la Universidade Federal de Santa Catarina (Brasil) y Coordinadora del Núcleo de Estudos e Pesquisas sobre Profissões e Instituições (NEPPI /UFSC/CNPq). Es especialista en biopolítica, riesgo, salud, saber médico, medicalización y saber psiquiátrico.

Esta entrevista fue realizada por la Lic. Sandra Ripoll en el mes de agosto de 2012, en la Escuela de Trabajo Social de la UNR y la versión que aquí se presenta ha sido revisada y aprobada para su publicación por la Dra. Mitjavila.

\section{SR: ¿Qué inquietudes te llevaron a estudiar la problemática del riesgo?}

MM: Mi interés por la problemática del riesgo nació de mis trabajos en torno a la medicalización del espacio social, o sea, de los procesos a través de los cuales la medicina amplía sus fronteras en términos de objetos de conocimiento y de objetos de intervención, para colonizar prácticamente todos los intersticios del espacio social. No hay nada que hoy en día permanezca fuera de la mirada y de la intervención médica; es el proceso progresivo de expansión del saber médico, que se incrementó, desde mi punto de vista, a partir del uso del riesgo como instrumento. ¿Por qué? Porque a partir del cálculo de probabilidades acerca de la eventualidad de que en un futuro se puedan experimentar daños a la salud, se justifican intervenciones médicas en los más diversos aspectos, que van desde la reproducción humana, pasando por la prevención de enfermedades cardiovasculares, hasta lo que tiene que ver con los comportamientos considerados moralmente indeseables, incluyendo no sólo conductas sino también actitudes y sentimientos. 


\section{SR: ¿Y cuáles son las principales perspectivas teóricas que desarrollan el concepto de riesgo?}

MM: Bueno, tal vez por su impacto a nivel internacional, por el enraizamiento que tuvieron tanto en la teoría social contemporánea como en los trabajos de muchísimos investigadores alrededor del mundo, yo diría que los teóricos de la modernización reflexiva son los que han tenido un impacto intelectual mayor en todo el mundo $Y$ en ese grupo podemos mencionar a Ulrich Beck con su clásico trabajo La sociedad del riesgo; a Anthony Giddens que vincula la problemática del riesgo con las transformaciones institucionales de la modernidad tardía; en cierta forma a Luhmann con su trabajo Sociología del riesgo; a la antropóloga Mary Douglas y, tal vez, a algunos otros. Más recientemente, tenemos algunos autores que son tributarios del legado foucaultiano, y que introducen la problemática del riesgo en los trabajos sobre la gubernamentalidad como problema contemporáneo: Nikolas Rose, por ejemplo, o Paul Rabinow. Todos ellos tienen puntos de encuentro que contribuyen a generar consenso en torno a la idea de que la modernidad es una cultura de riesgo, a que el riesgo puede adquirir diversos significados y a que, generalmente, la moralización y la politización de los peligros suelen organizarse a través de discursos y de prácticas sobre riesgo.

\section{SR: Ahora bien, la perspectiva en la que vos lo desarrollás es justamente pensarlo como un dispositivo biopolítico, ¿no?}

MM: Sí.

\section{SR: Entonces, ¿qué contribuciones nos puede brindar pensarlo desde esa perspectiva?}

MM: Puede ser que esa perspectiva no resulte interesante para investigar todos los temas; no percibo una capacidad omnicomprensiva en una estrategia de investigación como esa. Lo que pude evaluar a lo largo de los últimos años fue que esa perspectiva de considerar al riesgo como un dispositivo biopolítico presenta algunas virtualidades para examinar ciertos aspectos de la vida social contemporánea. En primer lugar, que ha permitido examinar mejor -sin perder la idea de totalidad social- algunos fenómenos que en un primer momento no vinculamos a procesos macro sociales o macro políticos, y que indagaciones genealógicas (en el sentido foucaultiano) permitieron identificar su relevancia en ese sentido. Esas indagaciones han permitido establecer conexiones entre procesos muy localizados -como sería el caso de los inherentes a la creación de bancos de datos po- 
blacionales informatizados- y las profundas y recientes transformaciones de las tecnologías políticas de gestión de lo social. Se trata de modalidades de gestión de lo social que se vienen enraizando en áreas como las vinculadas a la gestión biopolítica de la pobreza, pero igualmente en otras que tienen que ver con la reproducción biológica y social de la población; con la gestión de la criminalidad y con el área de la salud mental. Todas esas son áreas en las que ese tipo de perspectiva se ha mostrado productiva.

\section{SR: ¿Qué contribuciones pueden hacerle los estudios sobre riesgo a las intervenciones de los/as trabajadores/as sociales?}

MM: Posiblemente muchas más de las que yo puedo vislumbrar en este momento. Por un lado, los discursos y las prácticas organizadas en torno a la idea de riesgo operan en el corazón de las instituciones modernas. Por otro lado, los/as trabajadores/as sociales desarrollan sus prácticas en el corazón de las instituciones modernas; por lo tanto, podríamos decir que la actuación profesional del Trabajo Social ocurre independientemente de que el/la profesional lo perciba o de que lo desee. En la medida que ese ejercicio profesional transcurre al interior del funcionamiento de estos dispositivos el trabajo profesional se convierte en parte co-constitutiva del funcionamiento de los dispositivos biopolíticos. Además, el/la trabajador/a social debería ser un/a analista competente de la realidad social, incluyendo un análisis del funcionamiento de estos dispositivos, por lo cual determinados insights deberían ocurrir en el campo del Trabajo Social con relación al funcionamiento de los dispositivos y al papel de conocimiento científico y técnico en el desarrollo de esas tecnologías biopolíticas, las cuales exigen, en la mayor parte de los casos, que los/as trabajadores/as sociales participen en los procesos de alimentación de nuevos campos documentales en los cuales se registran informaciones sobre los individuos y nuevos campos documentales en los cuales se analizan perfiles poblacionales. Creo que representa más bien una oportunidad, un desafío para que el Trabajo Social examine el papel de sus prácticas profesionales en el desarrollo de las nuevas tecnologías biopolíticas.

\section{SR: En uno de sus trabajos ha planteado la noción de "carácter neoparaju- rídico". ¿Podría desarrollar esa idea?}

MM: Es una observación puntual y que está planteada más en términos hipotéticos que de constatación empírica y sin ninguna pretensión de generalización para el Trabajo Social en todo tiempo y lugar. Esa observación fue realizada a partir del desarrollo de algunos trabajos de investigación, sobre el papel del Tra- 
bajo Social en el campo sociojuridico en Brasil. Por ende, ese tipo de enunciados se restringe a las realidades que yo he podido observar y que responden a las peculiaridades de la trayectoria histórica de esta profesión en Brasil y en ese campo particular. En el campo jurídico ocurre que, cuando desarrollamos prácticas profesionales, esas prácticas siempre se edifican a partir de las lentes conceptuales que la profesión como colectivo y cada profesional utiliza. Sería correcto decir que o uno/a o bien usa la teoría social como lente para comprender la realidad -en este caso el tipo de realidad que se presenta en el campo jurídico- o usa otras cosas, la propia ideología, la religión, lo que fuere. Lo que ocurre muchas veces es que, en ausencia de marcos de referencia teóricos oriundos de las ciencias sociales, lo que pasa a oficiar de lente teórica es el discurso institucional. Entonces, por un camino que no es el camino que se experimentaba en los inicios de la profesión, sino por un nuevo rumbo, se puede volver a experimentar una suerte de subordinación, o la adopción de un carácter para-jurídico del Trabajo Social en lo que hace a sus fundamentos teóricos y, de ahí, la idea de neoparajudicialización. En el caso de Brasil estamos delante de una profesión que cuenta con una ley que la reglamenta, con organizaciones corporativas de la propia categoría que aseguran el cumplimiento de las condiciones -por lo menos del punto de vista formal- de autonomía técnica. Pero para que esa autonomía técnica ocurra debe existir una autonomía teórica y eso no necesariamente es lo que vemos...

\section{SR: Se lo preguntamos porque encontramos cierto correlato con nuestras prácticas. Finalmente y en este sentido, ¿qué contribuciones cree que se pueden hacer desde la formación académica para generar esa autonomía profesional?}

MM: Insisto en que no se puede generalizar y que la realidad de Brasil es una realidad muy diferente de la realidad, por ejemplo, de Argentina y de Uruguay que entre sí se encuentran más próximas. Con respecto a Brasil, pienso que lo que ocurre es esa especie de falta de marcos de referencia teóricos para la intervención profesional. Buena parte de la formación en los cursos de grado no proporciona lentes para analizar el tipo de fenómeno con el cual se trabaja en el ejercicio profesional. No estoy diciendo que los contenidos actuales no sirvan, sino que faltan contenidos que puedan ofrecer herramientas para un posicionamiento crítico y competente de los/as profesionales delante de los desafíos que presenta la realidad en la cual van a actuar. Esto supone, por lo menos, que la formación en los cursos de grado incorpore otras perspectivas teóricas, que hablen de esos fenómenos con los que se va a trabajar. Y no me refiero a líneas teóricas específicas, sino a que, independientemente de cuál sea la línea teórica, 
es preciso que se incorporen, tanto a la producción de conocimiento como a la formación de los cuadros profesionales, perspectivas de análisis y herramientas conceptuales y metodológicas en torno a asuntos con los cuales el/la trabajador/ a social estará obligado/a trabajar. Y esto hoy todavía no ocurre, por lo menos de una manera muy nítida en Brasil.

SR: Muchas gracias. 